\title{
Implementing an Energy Aware Issues in MANET by Designing Efficient Routing Protocol
}

\author{
Shivashankar \\ Dr. Ambedkar Institute of Technology \\ Bangalore-560056 \\ India
}

\author{
B.Sivakumar \\ Dr.A.I.T \\ Bangalore-56 \\ India
}

\author{
Dr.G.Varaprasad \\ B.M.S.C.E. \\ Bangalore-19 \\ India
}

\author{
Jayanthi.G \\ Dr.A.I.T \\ Bangalore-56 \\ India
}

\begin{abstract}
Mobile Ad Hoc Networks (MANETs) are multihop wireless networks in which all nodes cooperatively maintain network connectivity. In such a multihop wireless network, every node may be required to perform routing in order to achieve end to end communication among nodes. These networks are energy constrained as most ad hoc mobile nodes today operate with limited battery power. Hence, it is important to minimize the energy consumption of the entire network in order to maximize the lifetime of ad hoc networks. Since the emergence of mobile computing, reducing energy consumption of battery operated computing devices has become a very active research area. The widespread popularity of mobile computing devices, such as laptops, handheld devices and cell phones, motivates this research area. These nodes need to be energy conserved to maximize the battery life. Thus, development of energy efficient routing protocols is needed due to the limited battery power of all nodes. In this paper, we have considered two reactive protocols such as DSR and modified DSR (Efficient Power Aware Routing, EPAR) for MANETs and evaluated the energy performance metrics in all the four modes (transmitting, receiving, idle \& sleep) and the residual energy. Finally, by the observations we conclude that EPAR offers the best combination of energy consumption and network life time performance.
\end{abstract}

\section{Keywords}

MANET, multihop, energy consumption, DSR, Residual energy.

\section{INTRODUCTION}

One of the basic characteristics of a MANET is the multi-hop connection [1], in which mobile nodes cooperate to relay traffic to the distant destination node that would otherwise be out of direct communication range. Therefore, nodes in MANET serve not only as hosts [2], but also as routers. The multi-hop connection can also increase network capacity and decrease the energy consumption for transmission. However, due to the frequently changing network topology and limited resources of energy and wireless bandwidth, routing in MANET is an extremely challenging task. Basically, the routing protocol which chooses the best route between the source and destination nodes to fulfill the multihop transmission is called single path routing [3]. In cases of highly dynamic network topology and strictly limited resources, however, single path routing is not the best solution. Multipath routing protocols are then introduced[11], which provides redundant and alternative routes to assure successful data packet transmission and, at the same time, reduce the key relay nodes' power consumption, alleviating the network partitioning problem caused by the energy exhaustion of these nodes.
An Energy Aware link cache [4],[5],[10] is used to store the minimum transmits powers of the links in the route. The energy cost of the link is computed by a cost function which combines minimum transmits power for the link and the packet size. The minimum energy cost route is selected by applying Dijkstra's algorithm. Simulation results show that EPAR outperforms the traditional multipath routing protocol in providing longer network lifetime and lower energy consumption per bit of information delivered. In addition, as in other multipath routing protocols, it reduces the end-to-end delay [6] and improves the volume of packets delivered. Depending on the amount of network traffic [15],[16] and number of flows, the routing protocols could be chosen. When there is congestion in the network due to heavy traffic, in general case, a reactive protocol is preferable. Sometimes the size of the network might be a major considerable point.

\section{SOME OF THE RELATED RESEARCH WORKS}

Table-Driven routing protocols (Proactive): These protocols are also called as proactive protocols since they maintain the routing information even before it is needed [1]. Each and every node in the network maintains routing information to every other node in the network. Routes information is generally kept in the routing tables and is periodically updated as the network topology changes. Many of these routing protocols come from the link-state routing [8]. There exist some differences between the protocols that come under this category depending on the routing information being updated in each routing table. Furthermore, these routing protocols maintain different number of tables. The proactive protocols are not suitable for larger networks, as they need to maintain node entries for each and every node in the routing table of every node. This causes more overhead in the routing table leading to consumption of more bandwidth. (e.g.DSDV).

On Demand routing protocols (Reactive): These protocols are also called reactive protocols since they don't maintain routing information or routing activity at the network nodes if there is no communication. If a node wants to send a packet to another node then this protocol searches for the route in an on-demand manner and establishes the connection in order to transmit and receive the packet [5],[12]. The route discovery usually occurs by flooding the route request packets throughout the network. (e.g. DSR, TORA, AODV).

Many research efforts have been devoted for developing power aware routing protocols. Different approaches can be applied to achieve the target [16]. Transmission power control and load distribution are two approaches to minimize the active communication energy, and sleep/power-down mode is used to minimize energy during inactivity. 
Some research proposals, which are based on transmission power control approach, are discussed in [17-18]. Flow argumentation Routing (FAR) [17] which assumes a static network and finds the optimal routing path for a given sourcedestination pair that minimizes the sum of link costs along the path. Power aware Localized Routing (PLR) [17] is a localized, fully distributed energy aware routing algorithm but it assumes that a source node has the location information of its neighbors and the destination and Minimum Energy Routing (MER) [18] addresses issues like obtaining accurate power information, associated overheads, maintenance of the minimum energy routes in the presence of mobility and implements the transmission power control mechanism in DSR and IEEE 802.11 MAC protocol.

\section{ANALYSIS OF THE ENERGY ISSUE IN MANET}

\subsection{Basic Energy Model}

In this section, we present the energy model we use in this paper. Energy aware protocols consider transmit power, energy per packet, and remaining battery power; often with the goal of assigning a link-cost metric in a weighted shortestpath routing. In this work, we take a similar approach, considering the energy per packet.

The amount of energy spent in transmitting and receiving the packets is calculated by using the following equations:

Energy $_{\mathrm{tx}}=(330 *$ PacketSize $) / 2 * 10^{6}$ and

Energy $_{\mathrm{rx}}=(230 *$ PacketSize $) / 2 * 10^{6}$, where packet size is specified in bits.

The he energy consumed for one packet is given as:

$$
E=\sum_{i=1}^{k-1} T\left(m_{i}, m_{i+1}\right)
$$

where, $m_{i}$ to $m_{k}$ are nodes in the route while $T$ denotes the energy consumed in transmitting and receiving a packet over one hop. Then, minimum $\mathrm{E}$ for all packets is taken. However, this metric suffers a drawback as the nodes tend to have widely differing energy consumption profiles resulting in early death for some nodes. The energy expended in sending a data-packet of size D bytes over a given link can be modeled as

$$
E=X_{1} D+X_{2}
$$

We propose a similar model in our study to describe the per packet energy consumption. In this model,

$$
\begin{aligned}
& \mathrm{X}_{1}=\frac{\left(P_{t}^{\text {packet }}+P^{\text {back }}\right)}{R} \\
& \mathrm{X}_{2}=\frac{P_{t}^{M A C} D^{M A C}+P_{t}^{\text {packet }} D^{\text {headr }}}{R}+E^{\text {back }}
\end{aligned}
$$

where $\mathrm{P}^{\text {back }}$ and $\mathrm{E}^{\text {back }}$ are the background power and energy used in sending the data- packet, $\mathrm{P}_{\mathrm{t}}^{\text {MAC }}$ is the power at which the MAC packets are transmitted, $\mathrm{D}^{\mathrm{MAC}}$ is the size of the MAC packets, $D^{\text {header }}$ is the size of the data-packet trailer and header, $\mathrm{P}_{\mathrm{t}}{ }^{\text {packet }}$ is the power at which the data packet is transmitted and $\mathrm{R}$ is the transmission rate. In order to simplify our analysis, we assume $\mathrm{P}^{\text {back }}, \mathrm{D}^{\text {header }}$ and $\mathrm{P}_{\mathrm{t}}^{\mathrm{MAC}}$ are zero in this study.

\subsection{Power Decreases Rapidly with Increasing Distance}

If all the mobile nodes have energy model on at all time, the ongoing transmission is overheard by all the neighbors of the transmitter, which frequently happens in ad hoc net- works. In this scene, the neighboring nodes have to monitor the channel and consume power even though the packets are not directed to them. A large amount of energy is consumed unnecessarily in this case. Take a simple example: If a transmitter T has $n$ neighbors, then the transmit energy needed for a $m$-packet transmission is $m *\left(E_{\text {trans }}\right.$ (transmitting energy per packet $)+E_{r e c v}($ receiving energy per packet). However, since the transmission may be heard by all its neighbors, the actual power will be $m^{*}\left(E_{\text {trans }}+n^{*} E_{\text {recv }}\right)$. Obviously, it produces energy waste of $m *(n-1) * E_{\text {recv }}$. Based on this observation, a new kind of power conservation mechanism is proposed in which some nodes are allowed to stay in doze/sleep state when they are not actively transmitting, receiving, or waiting for a channel. Obviously, this power of mechanism can save battery power in all the mobile nodes of MANET.

\subsection{Minimum Energy Route Discovery}

Consider a case as shown in figures 1-4 (Minimum Power Route Discovery in EPAR to select the minimum power route) and 5-8 (Minimum Power Route Discovery in DSR to select the minimum power route) where there are 4 nodes A, $\mathrm{B}, \mathrm{C}$ and $\mathrm{D}$ in a straight line. Assume the minimum energy route from node $\mathrm{A}$ to node $\mathrm{D}$ is the multi-hop route A-B-C-D. The minimum energy routes in a network translate to multihop [6],[7],[8],[9] routes and the minimum energy routing protocol should be able to discover these minimum energy routes. The route discovery mechanisms of existing reactive protocols are similar in the way the route discovery is initiated. For finding a route from node A to node D, the mechanisms initiate a Route Request packet broadcast from node A.

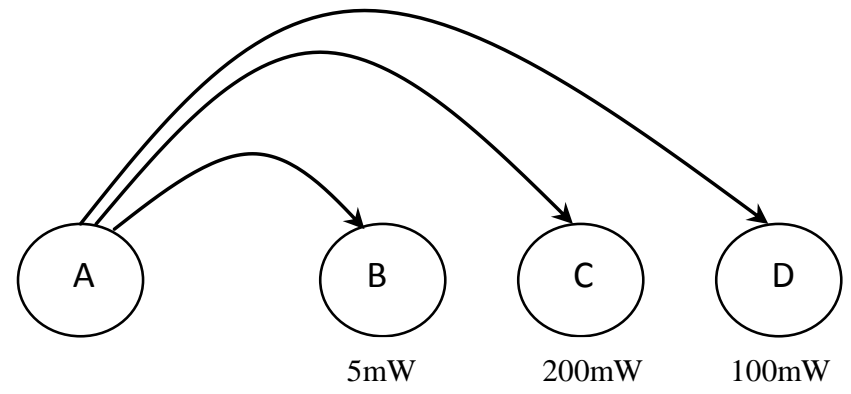

Fig. 1 Node A sends a route request and this route request packet is heard by $\mathrm{B}, \mathrm{C}$ and $\mathrm{D}$.

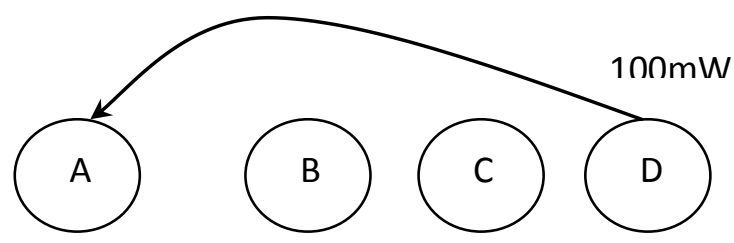

Fig. 2 Node $D$ sends a route reply A-D to node A. 


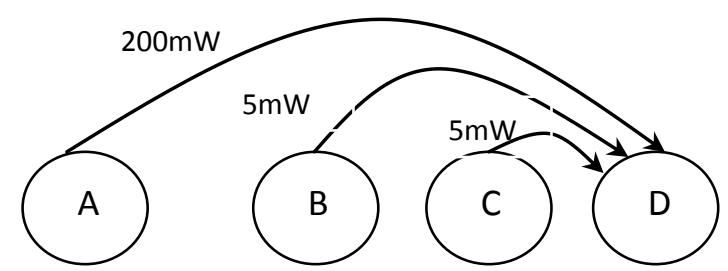

Fig. 3 Nodes $B$ and $C$ propagate this route request they hear from node $A$. However, node $C$ does not propagate the route request that $B$ broadcasts.

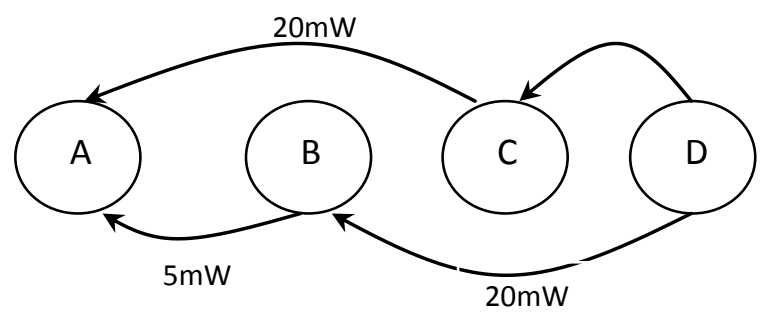

Fig. 4 Node D also sends the replies A-C-D and A-B-D to node $A$.

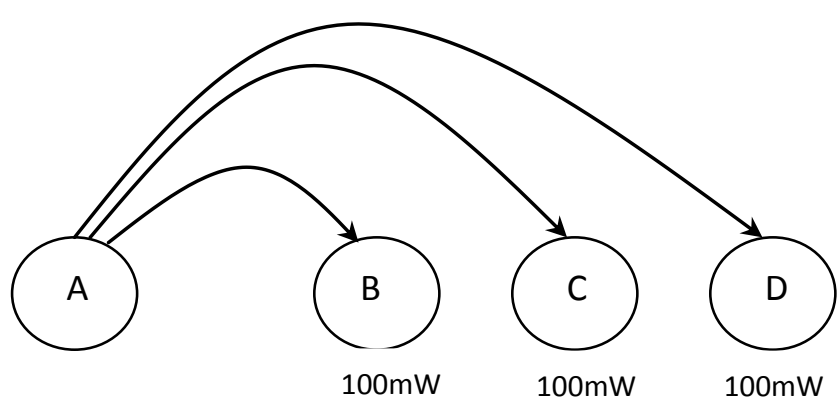

Fig. 5 Node A sends a route request and this route request packet is heard by $B, C$ and $D$.

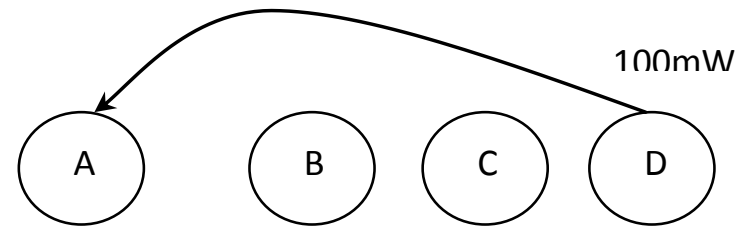

Fig. 6 Node $D$ sends a route reply A-D to node A.

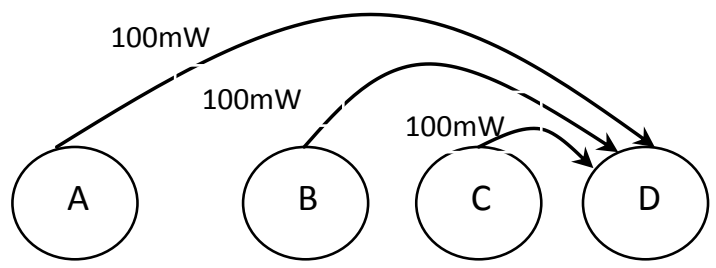

Fig. 7 Nodes $B$ and C propagate this route request they hear from node $A$. However, node $C$ does not propagate the route request that $B$ broadcasts

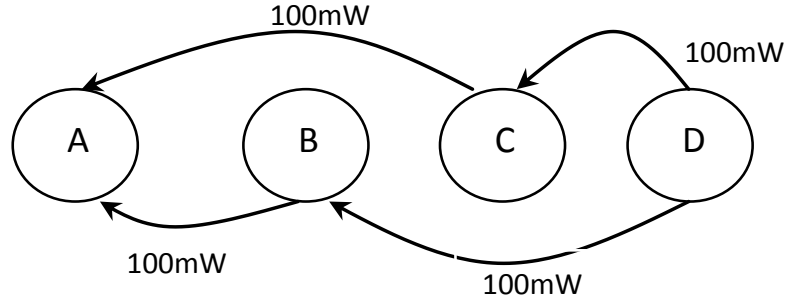

Fig. 8 Node D also sends the replies A-C-D and A-B-D to node $A$.

Assuming that this packet is heard by nodes B and C, both nodes rebroadcast the packet. The packets broadcasted by nodes $\mathrm{B}$ and $\mathrm{C}$ are heard by all nodes. However since node C has already broadcast the same request earlier, it ignores the request packet from node $\mathrm{B}$ and node $\mathrm{D}$ replies back to the requests it hears from nodes $B$ and $C$. Hence the reactive routes discovered by node $\mathrm{A}$ are A-D, A-B-D and A-C-D: The minimum energy route A-B-C-D is not discovered by the route discovery mechanism of the existing reactive routing protocols.

\subsection{Energy Aware Route Maintenance}

In the current version of reactive ad hoc routing protocols, route maintenance is carried out by the route error packets only when the links are broken. No route maintenance is done to indicate a change in the quality of a link. No mechanism updates the information about the changing energy cost requirements that occur on that route due to node mobility [13]. Even after the minimum energy cost routes are discovered, the changes in the energy costs of the links have to be tracked so that the energy expended is as close to the minimum value as possible. As nodes move closer together or further apart on a link the transmit power should decrease (to increase energy savings) or increase (to maintain the link) accordingly. Further, as the energy cost of a link rises due to the nodes moving apart, the route may no longer be the minimum energy cost route. Therefore, these changes in the energy cost need to be conveyed to the source node, so that it can choose other lower energy cost routes as needed. Hence a minimum energy routing protocol must have a mechanism for tracking the link energy cost changes. Mobility also causes the creation of new lower energy cost routes. Thus, to maintain minimum energy cost routing, additional route maintenance which goes beyond achieving basic connectivity is required. This feature also is not supported by existing versions of reactive routing protocols.

\section{DESIGN OF EPAR}

Existing versions of reactive ad hoc routing protocols do not possess most of the required features of a minimum energy routing protocol as demonstrated in the previous section. This section describes mechanisms for the easy implementation of these features in the routing logic of the existing reactive protocols.

\subsection{Minimum Energy Route Maintenance and Link Energy Cost Tracking}

In this paper each node can estimate the necessary transmission power and the link cost to one of its neighboring node once it receives RTS, CTS or broadcast packet from such node. And requires that each node adds the link cost to the receiver in the IP header as an IP option for each data packet it transmits, and monitors the data packets transmitted in its neighborhood. For each data packet transmitted, 
received, or overheard by the node, it will record the following information into a link cost table: (a) sender; (b) receiver; (c) link cost between the sender and the receiver; (d) source; (e) destination; (f) IP header ID; (g) the current time. Among these parameters, (a) and (b) can be obtained from the MAC header, while (c) to (f) can be obtained from the IP header. The information for a link will be kept only for a short time for accurate information and reducing storage overhead.

\subsection{Addition of new metrics into EPAR}

Two additional elements were added to the original implementation of EPAR Router. They were added to carry out transmit power control.

Set Power: This element carries out the functionality of setting the transmit power of the packet to the minimum transmit power value. In this section, we discuss the four experiments conducted to demonstrate a comparison of the protocols and highlight the energy efficient features of the EPAR protocol.

Max Power: This element carries out the functionality of setting the transmit power to the maximum value. These features include

- Energy based link cost with transmit power control,

- Energy based route selection,

- Energy based route discovery, and

- Energy based route maintenance.

This version of the EPAR protocol has transmit power control as its metric. To demonstrate the ability of EPAR to determine and adjust the link power due to varying path loss (i.e. distance), EPAR estimates the minimum transmit power required for that particular distance and uses the power value to transmit the packets to the destination.

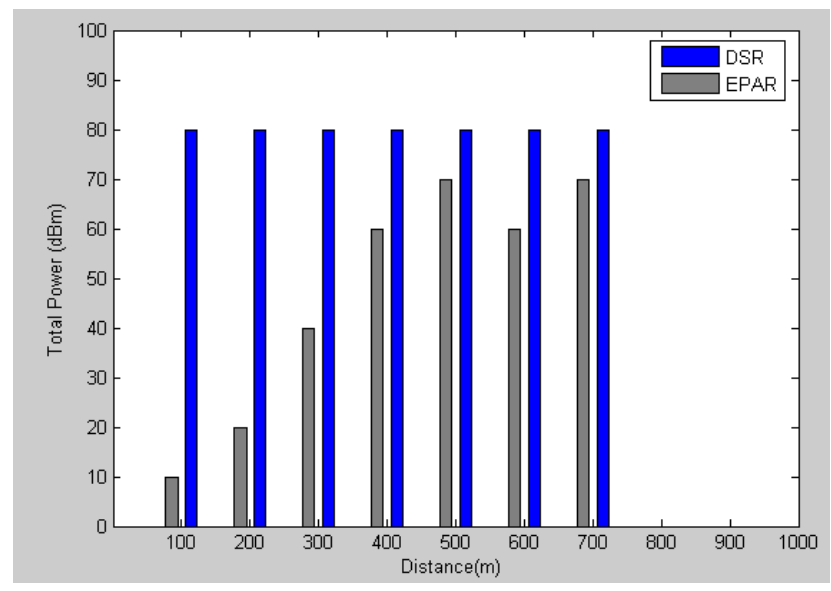

Fig 9: EPAR and DSR to Total Power vs. distance.

As shown in figure 9, the value of the transmit power increases as the distance $\mathrm{d}$ between the nodes increases. When the distance $\mathrm{d}$ is $100 \mathrm{~m}$, EPAR estimates the minimum transmit power required for the source node to communicate successfully with the destination to be $10 \mathrm{dBm}$ and achieves successful communication between the source and destination node. As the distance between the nodes is increased, EPAR realizes that the nodes can no longer communicate at a low power value and it estimates the value of the minimum transmit power required for successful communication. It does this for every value of $d$. As seen in the fig 9, the DSR protocol always transmits at $80 \mathrm{dBm}$ for all distances thus expending more energy compared to the EPAR protocol.

\section{IMPLEMENTATION}

By referring to figure 1-8 we observed that both protocols discover the routes A-D and A-B-C-D. DSR uses the minimum hop route A-D to route the packets and transmits them at $80 \mathrm{dBm}$. EPAR discovers that the route A-B-C-D is the minimum power path and uses this route to transmit packets.

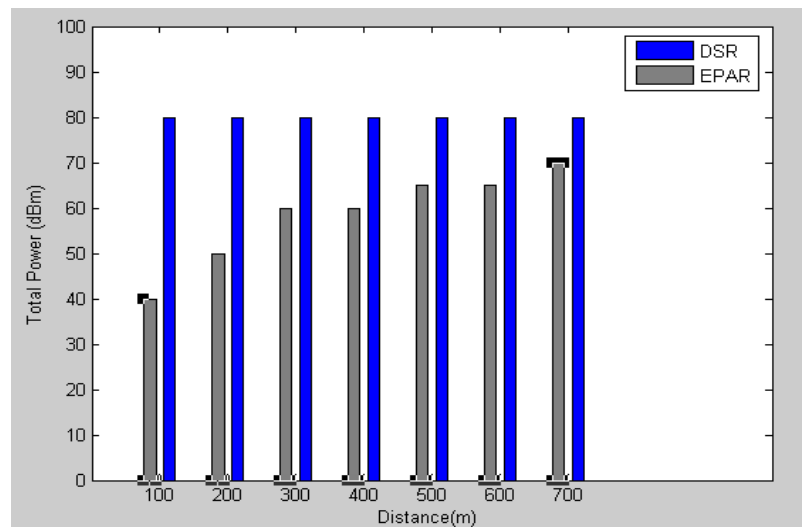

Fig 10: Minimum Power Route Choice: Comparison of the routes selected by DSR and EPAR.

As seen in figure 10 , as d increases the total transmit power used by EPAR to route packets increases. When d equals 100 $\mathrm{m}$, EPAR uses a total transmit power of around $40 \mathrm{dBm}$ to transmit packets from the source node A to destination node D via intermediate node $\mathrm{B}$ and $\mathrm{C}$. However, the DSR protocol uses $80 \mathrm{dBm}$ to carry out the same task using the direct hop route A-B-C-D. Similarly for the other two values of the distances, EPAR consumes less transmit power compared to DSR.

\subsection{Route Power Maintenance}

In this metric, we used source node A, intermediate node B and $\mathrm{C}$, and destination node $\mathrm{D}$. The distance between the nodes $\mathrm{A}$ and $\mathrm{D}$ were fixed during the course of the experiment and the nodes were placed along a straight line. As shown in fig 5-8, the intermediate node $\mathrm{b}$ was placed along a line perpendicular to the line joining nodes $A$ and D. Initially the router on node B was turned off and to bring about significant energy savings as compared to the DSR protocol. The experiments described in table 2 shows the power budget for the transmission of a single data packet for the simulation with the greatest overhead. To compare the power expended by each protocol, we assume equal packet lengths (the control packets are smaller than the data packets).

As seen from the table, despite the additional route discovery overhead, EPAR still consumes lower power than DSR starting with the first packet. Subsequent data packets transmitted using EPAR will result in further power savings as shown in Table 2. The ACK packets were transmitted at full power to ensure robust behavior of the protocol. However, if they are transmitted by adding a margin MACK that equals $9 \mathrm{dBm}$, the power per subsequent packet as shown in Table 2 will effectively reduce to $100 \mathrm{~mW}$ which is four times lower power than DSR. These power savings are achieved at the routing layer. To compute energy savings in transmission, we need to consider actual packet lengths for which these power savings obtained in EPAR will translate to transmission energy savings. 


\subsection{EPAR Protocol with Packet Energy \\ Metric}

The energy cost of a link is modeled in a more realistic manner and hence we introduced a new energy aware routing metric that was based on the model. The tests carried out in this section to demonstrate the behavior of the EPAR protocol to route packets based on the energy cost of the link. This cost depends on the packet size, minimum transmit power of the link, and the background energy associated with that link. For small packets, this energy cost is dominated by the background energy of the link whereas transmission energy dominates for large packets. Hence to conserve energy while routing packets, it follows that smaller packets should be routed using the minimum hop route and large packets should be routed using the multihop path.

\subsection{Minimum Cost Route Choice}

We used source node A, intermediate node B and C, destination node $\mathrm{D}$. These nodes were placed along a straight line. The position of node B was fixed and was placed an equi-distance, $\mathrm{D}$, from the source and destination node. We varied the positions of nodes $\mathrm{A}$ and $\mathrm{D}$ along this line such that they were equi-distant from the intermediate node B all through the experiment. At each position, ping packets of sizes 256, 512 and 1024 bytes were initiated. Routers on nodes A-B-C-D are simultaneously started. We observed the behavior of EPAR to select different routes depending on the distance between $\mathrm{A}$ and $\mathrm{D}$, and the packet size. Figure 4 depicts the minimum transmit power required for a particular route, and total energy per packet involved to route a packet from source node $A$ to destination node $C$ for varying packet sizes. EPAR uses the multihop route A-B-C-D to route. As observed from figure 11, EPAR selects routes depending on distance between the source and destination node and packet size. When the distance between the source and destination is $100 \mathrm{~m}$, packets of length 1024 and 512 bytes. EPAR computes that the energy required to route packets is $30 \mathrm{~J}$ and $20 \mathrm{~J}$ respectively. EPAR computes that the multi-hop route is cheaper as compared to the direct route a-b with a cost of 117 J. However, it uses the direct route A-B-C-D when the packet size is reduced to 256 bytes.

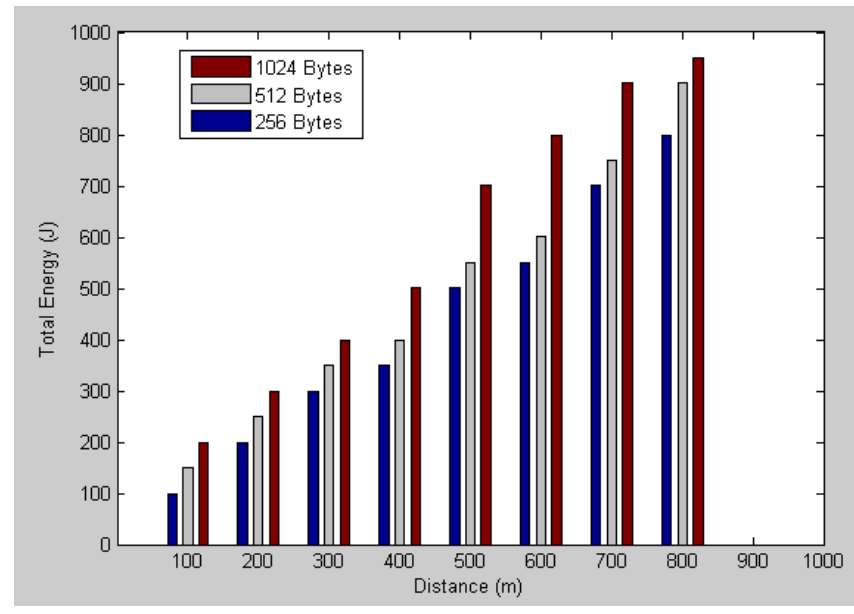

Fig 11: Three Nodes choice: comparison of Energy cost based on packet size.

When the distance between node a and $\mathrm{c}$ is increased to 100 $\mathrm{m}$, EPAR once again uses the multi-hop route A-B-C-D to route packets of length 1400 bytes with total cost of $50 \mathrm{~J}$ and $30 \mathrm{~J}$ respectively. EPAR realizes that the multihop routes are cheaper than the direct hop route. However, the 256 byte packets are always routed using the direct route A-D. Although EPAR does have the option of routing larger packets via the route $a-b$, it chooses the multi-hop route A-D since the cost as seen from the figure 11 for routing larger packets over large distance using the multi-hop route is less than the cost to route packets using the minimum hop route. However, for smaller packets, the multihop route cost proves to be more expensive than the minimum hop route, hence small packets are routed using minimum hop routes.

\section{SIMULATION RESULT AND DISCUSSIONS}

There are two ways to implement the new algorithm in Network Simulator (NS-2.33).

1. Because the new algorithms are based on the DSR protocol, it is possible to modify directly the DSR protocol and do the tests with the modified version. Or

2. Add a new protocol in NS-2.33.

We have chosen the second solution, because it is more practical to have both the DSR and new protocols in the same version of NS-2.33 for testing purposes. Indeed, it is easier to compare the performance of the two protocols. So, we copied the DSR folder and create new one: the EPAR protocol for the new algorithm. These extensions include the modeling of an IEEE 802.11/MAC. Table 1 shows the simulation parameters used in the network setup. The metrics of the energy efficiency and network performance are studied.

Table 1: Simulation parameters.

\begin{tabular}{|l|l|}
\hline Number of nodes & 120 \\
\hline Area size & $2000 \times 2000$ \\
\hline Mobility model & Random Way point \\
\hline Traffic type & CBR \\
\hline Packet size & $256,512 \& 1460$ Bytes \\
\hline Frequency & $1 \mathrm{Ghz}$ \\
\hline Channel capacity & $2 \mathrm{M} \mathrm{bps}$ \\
\hline Transmit power & $2.0 \mathrm{Mw}$ \\
\hline Receiver power & $2.0 \mathrm{Mw}$ \\
\hline Communication system & MAC/IEEE $802.11 \mathrm{G}$ \\
\hline Routing Protocols & DSR, EPAR \\
\hline
\end{tabular}

\subsection{Energy Efficiency Metrics}

We evaluate the energy efficiency with two metrics: network lifetime and energy dissipation per bit of data delivered. Firstly, we observe the variation of network lifetime while the data rate of the CBR flow is increased and under different mobility scenarios. 


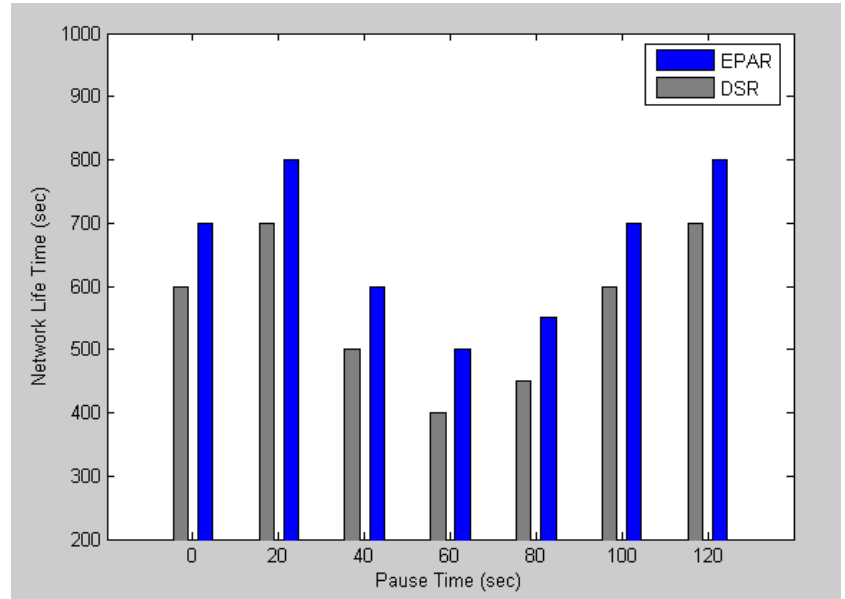

Fig 12: network Life Time (Mobility)

Note that in wireless ad hoc networks, especially in those with densely distributed odes, the death of the first node seldom leads to the total failure of the network. With the number of dead nodes increasing, the network is partitioned. Even with partitioning, end to end transmissions may still be feasible in each partition. Basically, we can argue that the network is alive if there exists at least one pair of adjacent nodes working, since they could transmit to each other and keep the network alive. So, the strict definition of the network lifetime is ambiguous. Taking into consideration of the statistical mean effect and the large number of repeated experiments under equivalent scenarios, we define the time when the first node in the network runs out of its energy as the network lifetime. Figure 12 shows the simulation results on network lifetime comparing EPAR and DSR under various traffic loads and different mobility scenarios. We can see that networks running EPAR live longer than those running DSR.

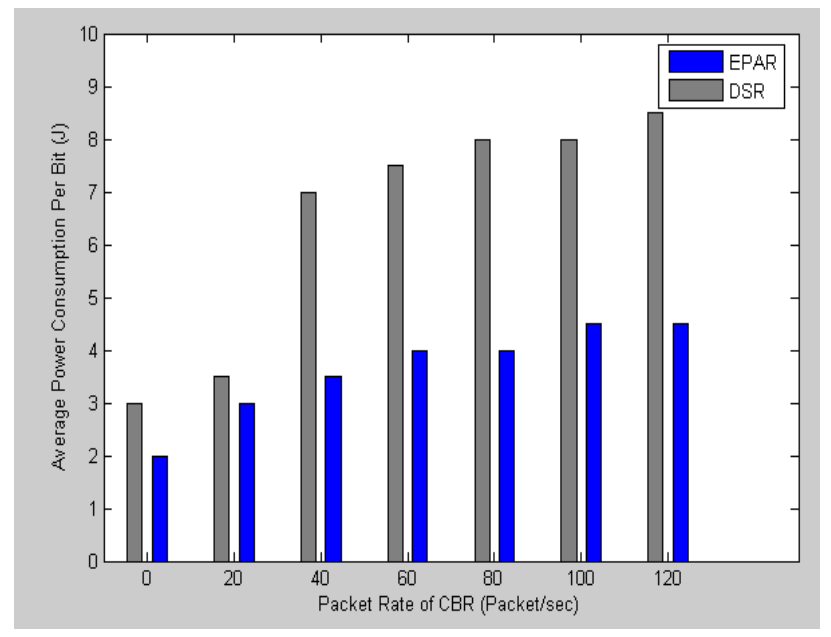

Fig 13: Average Energy Consumption per Bit delivered

Figure 13 demonstrates the average energy consumption per bit delivery. It is obtained by dividing the sum of the energy consumption of the network by the number of successfully delivered bits. The horizontal axis represents the traffic load and the result is again obtained under dynamic topology. We see that EPAR outperforms DSR by about 35\%-150\% under different traffic loads. Before the traffic load reaches 20 packets per second, the gain in average energy consumption from EPAR over the DSR is about $35 \%-50 \%$, which is mainly due to the benefit of power control in the MAC layer. Between 20 packets per second and 40 packets per second, there is a distinct increase in the average energy consumption for the DSR network, which is because 20 packets per second is the saturation point of DSR, i.e. the maximum load which can be accommodated in a single route. The excess packets inevitably introduce more collisions to the network, wasting more energy. EMRP chooses alternative routes, avoiding the heavily burdened nodes, thus alleviating the explosion in average energy consumption.

\subsection{Network Performance Calculation}

From figure 12 and 13, the network performance is evaluated with two metrics, namely, the volume of packets delivered and the end-to-end delay. Note that the volume of packets delivered is equivalent to the network throughput as a metric to evaluate the network's capacity of packet delivery in our simulation, because the data traffic scenarios are the same for the two networks running EPAR and DSR, respectively. The volume of packets delivered is the total number of CBR packets received by the intended receiver during the simulation. Ideally, it should increase linearly with the traffic load. But the nodes' limited energy and excessive collisions lead to a different behaviour.

Table 2: Energy Consumption calculation in DSR and EPAR protocol.

\begin{tabular}{|l|c|c|}
\hline Packet Type & $\begin{array}{c}\text { Power consumption } \\
\text { in DSR } \\
\text { (Number of packets } \\
\text { x Power transmitted } \\
\text { at }(\mathrm{mW}))\end{array}$ & $\begin{array}{c}\text { Power consumption in } \\
\text { EPAR } \\
\text { (Number of packets } \mathrm{x} \\
\text { Power transmitted at } \\
(\mathrm{mW}))\end{array}$ \\
\hline Route Request & $3 \times 100$ & $3 \times 100$ \\
\hline Route Reply & $2 \times(100+100)$ & $\begin{array}{c}1 \times(100+20)+ \\
1 \times(100+100)\end{array}$ \\
\hline Data Packet 1 & $1 \times(100+100)$ & $1 \times(120)$ \\
\hline ACK packet & $100+100$ & $100+100+100$ \\
\hline $\begin{array}{l}\text { Total Power } \\
\text { First packet }\end{array}$ & 1100 & 1040 \\
\hline $\begin{array}{l}\text { Power per } \\
\text { Subsequent } \\
\text { packet }\end{array}$ & $4 \times 100=400$ & $(3 \times 100+(20+5+5))$ \\
$=330$
\end{tabular}

\section{CONCLUSION}

This paper answers the research question whether a robust distributed energy conserving ad hoc routing protocol can be implemented by developing a cross-layered protocol that can conserve significant energy. We have successfully implemented the DSR protocol and modified it to develop a new protocol, namely the Efficient Power Aware Routing (EPAR) protocol. The EPAR protocol employs, link cost adjustments and transmit power control and minimum cost route discovery and maintenance to achieve energy efficiency. Experimental results demonstrate that the EPAR protocol results in a fifty to sixty percent of energy saving and this saving is obtained starting from the transmission of the first packet. Subsequent packets transmitted using the EPAR protocol will result in further energy savings. These savings are obtained at the routing layer by assuming an energy efficient MAC protocol with the selected background energy. The main objective of EPAR is to minimize the variance in 
the remaining energies of all the nodes and thereby prolong the network lifetime. In the future, we plan to investigate the nodes' behavior under different mobility scenarios and further improve the performance of EPAR by tuning the parameters of the protocol with large network. Finally, we also point out some of the overheads of using EPAR and show clearly when EPAR performs better and what are the factors that affect it.

\section{REFERENCES}

[1] S.R.Biradar, Hiren H.D.Sharma, Kalpana Sharma and Subir Kumar Sarkar, "Performance Comparison of Reactive Routing Protocols of MANETs Using Group Mobility Model", IEEE International Conference on Signal Processing Systems, pages 192-195, 2009.

[2] Suresh Kumar, R.K.Rathy and Diwakar Pandey, “Traffic Pattern Based Performance Comparison of Two Routing Protocols for Ad hoc Networks Using NS2", 2nd IEEE International Conference on Computer Science and Information Technology, 2009.

[3] Wang Lin-Zhu, Fang Ya-qin and Shan Min, "Performance Comparison of Two Routing Protocols for Ad Hoc Networks", WASE International Conference on Information Engineering, 2009.

[4] C.Mbarushimana and A.Shahrabi, "Comparative Study of Reactive and Proactive Routing Protocols Performance in Mobile Ad Hoc Networks", AINAWIEEE, 2007.

[5] D.Johnson, Y.Hu \& D.Maltz, "The Dynamic Source Routing Protocol(DSR) for Mobile" , RFC 4728, Feb 2007.

[6] UCB/LBNL/VINT Network Simulator, http://wwwmash.cs.berkeley.edu/ns/referred on March 2010.

[7] "The Network Simulator- ns-2", available at http://www.isi.edu/nsnam/ns/referred on march 2010.

[8] L.M.Feeney and M. Nilsson, "Investigating the Energy Consumption of a Wireless Interface in an $\mathrm{AD} \mathrm{Hoc}$ Networking Environment", Proceedings of IEEE INFOCOM 2001, Volume 3, Anchorage A.K. April 2001, pages 1548-1557.

[9] L.M.Freeney, “An Energy Consumption Model for Performance Analysis of Routing Protocols for Mobile
Ad hoc Networks", Mobile Networks and Applications, Volume 6, Issue 3, June 2001, Pages 239-249.

[10] A Safwat, H. Hassanein and H. Mouftah, "Energy aware Routing in MANETs: Analysis and Enhancements", Proceedings of The Fifth ACM International Workshop on Modeling, Analysis and Simulation of Wireless and Mobile Systems(MSWiM 2002) in conjunction with the ACM Mobile Computing and Networking Conference, Atlanta, Georgia,USA, pages 46-53.

[11] Harminder S.Bindra, Sunil K.Maakar and A.L.Sangal, "Performance Evaluation of Two Reactive Routing Protocols of MANET using Group Mobility Model", International Journal of Computer Science Issues, Vol.7, Issue 3, No.10, May 2010.

[12] M. Li, L. Zhang, V. O. K. Li, X. Shan and Y. Ren, "An Energy-Aware Multipath Routing Protocol for Mobile Ad Hoc Networks", Proceedings of the ACM SIGCOMM Asia, April 2005.

[13] Mohammed Bouhorma, H. Bentaouit and A. Boudhir "Performance Comparison of Ad Hoc Routing Protocols AODV and DSR", IEEE, 2009.

[14] Wang N-C, Huang Y-F, Chen J-C (2007) A stable weight-based on demand routing protocol for mobile ad hoc networks. Information Sciences: an International Journal, Volume 177, Issue 24:5522-5537

[15] G.Vijaya Kumar, Y.Vasudeva Reddyr,and Dr.M.Nagendra, "Current Research Work on Routing Protocols for MANET: A Literature Survey", International Journal on Computer Science and Engineering, Vol. 02, No. 03, 2010, pp. 706-713.

[16] Chang J-H, Tassiulas L, "Energy Conserving Routing in Wireless Ad-hoc Networks," Proc. IEEE International Conf. on Computer Communications (IEEE Infocom) 2000; pp 22-31

[17] Stojmenovic I, Lin X. "Power-Aware Localized Routing in Wireless Net-works," IEEE Trans. Parallel and Distributed Systems 2001; 12(11):1122-1133.

[18] Doshi S, Brown TX, "Minimum Energy Routing Schemes for a Wireless Ad Hoc Network," Proceedings of the Conference on Computer Communications (IEEE Infocom 2002), 2002. 